See the related article beginning on page 479 .

\section{The role of uncoupling protein 3 in human physiology}

\author{
W. Timothy Garvey \\ Division of Endocrinology, Diabetes, and Medical Genetics and Department of Medicine, \\ Medical University of South Carolina, and Ralph H. Johnson Veterans Affairs Medical Center, \\ Charleston, South Carolina, USA
}

J. Clin. Invest. 111:438-441 (2003). doi:10.1172/JCI200317835.

Obesity is simply understood as an imbalance between energy intake and expenditure in favor of weight accretion. However, the human biological interface between food consumption and energy dissipation results in broad individual differences in eating behavior, physical activity, and efficiency of fuel storage and metabolism. In particular, the basal metabolic rate, which accounts for the greatest portion of overall energy expenditure, can vary almost twofold among individuals. Classically, three major biochemical systems are believed to contribute to basal thermogenesis: futile cycles, $\mathrm{Na}^{+} / \mathrm{K}^{+}$ATPase activity, and mitochondrial proton leak. The latter is the most important quantitative contributor and can explain up to $50 \%$ of the basal metabolic rate (1). The molecular basis of mitochondrial proton leak is unclear, despite its importance in the understanding of energy balance and its potential as a therapeutic target for obesity treatment. The article by Hesselink and colleagues in this issue of the JCI (2) addresses whether uncoupling protein 3 contributes to mitochondrial proton leak in human skeletal muscle.

\footnotetext{
Address correspondence to: W. Timothy Garvey, Division of Endocrinology, Diabetes, and Medical Genetics, Clinical Science Building 816, Medical University of South Carolina, 96 Jonathan Lucas Street, Charleston, South Carolina 29425, USA. Phone: (843) 876-5372; Fax: (843) 876-5133; E-mail: garveywt@musc.edu.

Conflict of interest: The author has declared that no conflict of interest exists.

Nonstandard abbreviations used: brown adipose tissue (BAT); uncoupling protein 1 (UCP1).
}

\section{Mitochondrial respiration and oxidative phosphorylation}

The oxidation of fatty acids and pyruvate takes place in mitochondria, where energy is converted into ATP for use in cellular processes. Reducing equivalents are extracted from substrates and sequentially passed from electron donors (reductants) to acceptors (oxidants) along the mitochondrial respiratory chain to molecular oxygen. The electron transport system is located on the inner mitochondrial membrane, where oxidation steps are coupled by the transport chain to the extrusion of protons out of the matrix. This establishes an electrochemical potential difference across the inner membrane and a motive force for proton reentry through $\mathrm{F}_{1} \mathrm{~F}_{0}$-ATP synthase. ATP synthase captures the potential energy released upon proton reentry by converting ADP to ATP. In this manner, electron transport is coupled to oxidative phosphorylation. In a perfectly coupled system, protons only enter the mitochondrial matrix through ATP synthase in the presence of ADP; this form of respiration is classified as state 3 (i.e., $\mathrm{O}_{2}$ is consumed only in the presence of substrate and ADP). However, mitochondria can also be observed to use oxygen even in the absence of ADP, which occurs when protons leak back into the matrix via a mechanism that does not involve $\mathrm{F}_{1} \mathrm{~F}_{0}$-ATPase. This proton leak reduces the proton gradient driving ATP formation and uncouples respiration from oxidative phosphorylation. Oxygen utilization in the absence of ADP or in totally uncoupled mitochondria is referred to as state 4 respiration.

\section{Uncoupling proteins}

The mechanisms mediating ATPaseindependent proton leak have not been identified, except in mammalian brown adipose tissue (BAT). BAT is rich in mitochondria and lipid droplets and is a major source of nonshivering thermogenesis, used by most mammals to resist cold. This function is mediated by uncoupling protein 1 (UCP1) (previously known as uncoupling protein or thermogenin), first cloned in 1985 (3). UCP1 localizes to the mitochondrial inner membrane and dissipates the transmembrane potential by transporting protons from the intermembrane space back into the matrix. This reduces the proton motive force that drives ATP formation, and respiration in the uncoupled mitochondria proceeds, releasing fuel energy only as heat. In humans and other large mammals, BAT disappears after infancy, and there is minimal or no detectable UCP1 expression in adults. However, even in the absence of UCP1, there is a finite proton leak across the inner membrane that cannot be explained by simple diffusion (4). This led investigators to search for additional uncoupling proteins with broader tissue expression, and two other members of the uncoupling protein family were identified in 1997. The genes encoding human UCP2 (5, $6)$ and UCP3 $(7,8)$ lie in close proximity to each other on chromosome $11 \mathrm{q} 13$ and share $55 \%$ and $57 \%$ amino acid identity with UCP1, respectively. UCP2 mRNA is widely expressed in multiple tissues, while UCP3 exhibits more limited tissue-specific expression confined to skeletal muscle and brown fat tissue. UCPs have similar predicted topology consisting of six transmembrane regions linked by polar loops and are localized to the inner mitochondrial membrane. More recently, two additional UCP-like genes, UCP4 and UCP5/brain mitochondrial carrier protein-1, have been identified, which are expressed in the brain and have relatively lower amino acid identity with UCP1 (30-40\%).

Because of their homology with UCP1 and expression in adult tissues, UCP2 and UCP3 immediately were considered as attractive candidates for proteins involved in energy expenditure. When 


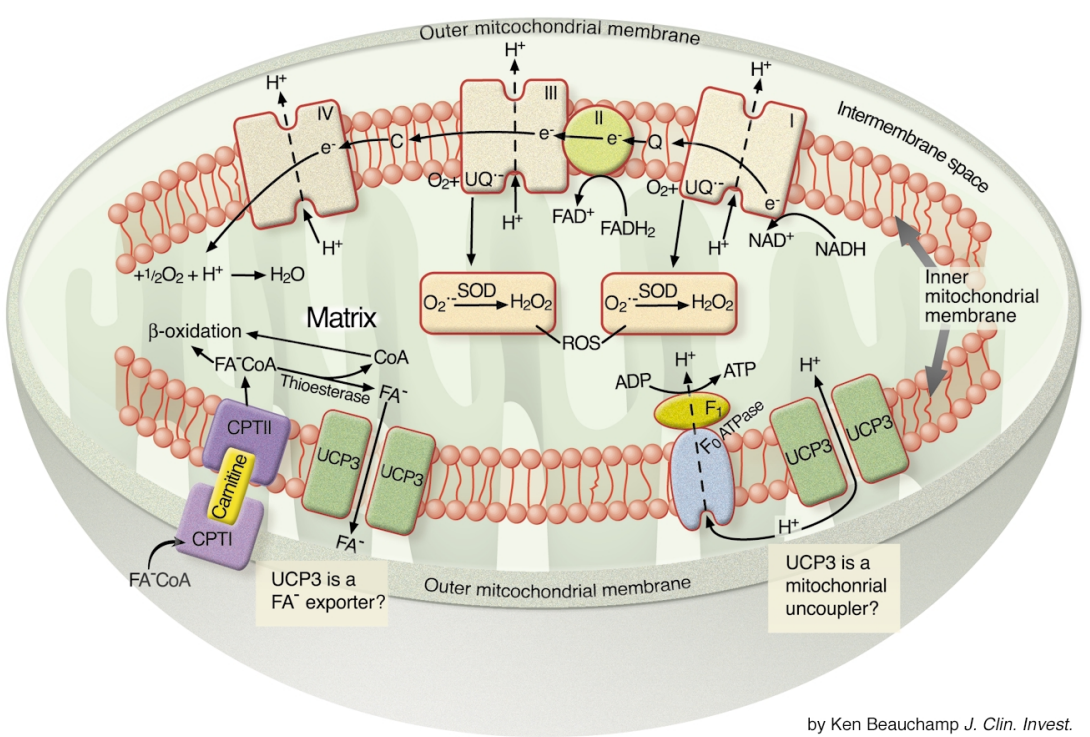

Figure 1

Uncoupling proteins and mitochondrial function. The figure shows key proteins in the inner mitochondrial membrane involved in mitochondrial respiration, oxidative phosphorylation, uncoupling, and import of long-chain acyl-CoA molecules. Two potential roles for UCP3 function are illustrated. (right-hand side) UCP3 functions as an uncoupler by acting as a channel for proton entry into the matrix, which dissipates the transmembrane potential generated by respiratory chain complexes I through IV. This reduces the motive force for proton entry via the $F_{1} F_{0}$-ATPase, which catalyzes ATP synthesis, and, in effect, uncouples respiration from oxidative phosphorylation. Substrate oxidation proceeds via transfer of electrons from donors (reductants) to acceptors (oxidants) along the respiratory chain to water, releasing energy as heat. Another consequence is a reduction in reactive oxygen species formation, since these species are generated under conditions of high transmembrane potential and electron flow. (left-hand side) In another scenario, UCP3 acts as an exporter of fatty acid anions (FA-). This could facilitate fatty acid oxidation and explain experimental observations linking regulation of UCP3 expression and genetic variation with effects on fat oxidation. Under conditions of high fatty acid flux into mitochondria via carnitine palmitoyltransferase 1 (CPT1), excessive accumulation of long chain acyl-CoA molecules would be harmful to membranes and sequester $\mathrm{CoA}$, thereby impairing fat oxidation. To prevent these events, upregulation of mitochondrial thioesterase cleaves the acyl-CoA allowing export of the fatty acid anion via UCP3. Reuptake of a neutral fatty acid could deliver the proton (plus fatty acid anion) back into the matrix resulting in uncoupling; however, the fatty acid export function would not necessarily depend upon an uncoupling action for UCP3. e-, electron; I, Complex I or NADH-ubiquinone oxidoreductase; II, Complex II or succinate ubiquinone oxidoreductase; III, Complex III or ubiquinol-cytochrome c oxidoreductase; IV, Complex IV or cytochrome c oxidase; Q, coenzyme Q or ubiquinone; c, cytochrome

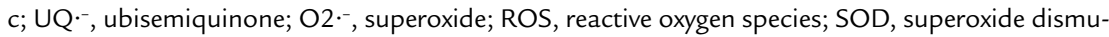
tase; $F_{1}$ is the water-soluble nucleotide-binding complex and $F_{0}$ is the hydrophobic transmembrane complex that together comprise the $\mathrm{F}_{1} \mathrm{~F}_{0}$-ATPase.

expressed in yeast and mammalian cell lines, both UCP2 and UCP3, like UCP1, were shown to act as uncouplers, as evidenced by a decrease in mitochondrial membrane potential and increases in mitochondrial state 4 respiration, whole cell $\mathrm{O}_{2}$ consumption, and heat production. In addition, UCP1, UCP2, and UPC 3 shared in common the ability to be regulated by factors important in energy balance including $\beta 3$-adrenoreceptor agonists, triiodothyronine, food intake, and cold exposure (although cold exerted more modest effects on UCP2 and UCP3 than on UCP1). However, the paradigm began to unravel increase in the ATP/ADP ratio, and a decrease in state 4 respiration, consistent with an uncoupling effect for UCP3 when present in the wild-type mice. Transgenic mice hyperexpressing UCP3 were characterized by reduced body weight, despite being hyperphagic, by increased resting $\mathrm{O}_{2}$ consumption, and by increased muscle temperature but not core temperature. At the same time, isolated mitochondria exhibited decreased transmembrane potential and increased state 4 respiration. These results in transgenic mice were more consistent with a role for UCP3 as a mitochondrial uncoupler.

\section{Importance of human studies}

Hesselink et al. (2) have studied the capacity of UCP3 to act as an uncoupler in human skeletal muscle following a diet-induced increase in UCP3 expression. Healthy male volunteers expressed 44\% more UCP3 protein in skeletal muscle while consuming a high-fat diet than while consuming a low-fat diet. The investigators assessed mitochondrial function in vivo by measuring phosphocreatine resynthesis following anoxic muscle contractions. To permit rapid bursts of muscle contraction, highenergy phosphate bonds are stored as phosphocreatine, which is formed by transfer of phosphate from ATP to creatine catalyzed by creatine kinase. The rate of phosphocreatine resynthesis reflects ATP synthetic rates via mitochondrial $\mathrm{F}_{1} \mathrm{~F}_{0}$-ATPase, which in turn is affected by the extent of mitochondrial uncoupling. The authors observed similar time courses of phosphocreatine repletion in the high-fat and low-fat fed subgroups, indicating that the physiological upregulation of UCP3 with high-fat feeding did not affect the mitochondrial proton leak in vivo. The authors also found that relative amounts of free carnitine and acylcarnitine were similar in both subgroups, verifying that there were no differences in substrate availability (at least from longchain fatty acids). The authors were able to reject their initial hypothesis and conclude that UCP 3 does not act as a mitochondrial uncoupler in human muscle under these physiological conditions.

The paper is instructive for several reasons. First, the authors have quantified UCP3 expression at the protein 
level using well-characterized and specific affinity-purified antibodies. The literature to date has relied heavily on measurements of mRNA to study UCP2 and UCP3 expression, since available antibodies have lacked specificity. Second, the authors have assessed mitochondrial uncoupling in vivo following a diet-induced physiological perturbation of UCP3 levels. In knock-out mice, evidence that UCP2 or UCP3 functions as an uncoupler has derived from experiments performed in isolated mitochondria ex vivo, not in intact tissues. The lack of effects on energy metabolism and body weight in these mice would suggest that the uncoupling in isolated mitochondria either does not occur in vivo or is not physiologically significant. In addition, Brand and his colleagues have recently found that uncoupling in the presence of supraphysiological UCP3 hyperexpression, at the high levels obtained in transfected yeast and in UCP3 transgenic mice, was induced by artifact and did not represent properties of the native protein (11). This observation makes it difficult to derive meaningful physiological information from the study of the transgenic mice (12), which hyperexpressed UCP3 more than 50-fold above the level in wild-type mice (11). The emerging picture is that accurate physiological information can most confidently be obtained when mitochondrial uncoupling is assessed in vivo within the physiological range of UCP regulation.

Third, the authors studied human beings. While transgenic mice experiments have been highly prized by firstline biomedical journals, experiments involving human physiology have been relatively undervalued in my opinion. Particularly in the area of energy expenditure, it is problematic to extrapolate data in transgenic mice to human physiology since adult humans do not have brown fat and heat production per kg body weight is much lower in humans than in rodents. This lesson was learned in the case of leptin, which exerts major effects on body temperature in rodents, while this physiological action is markedly attenuated or lacking in humans $(13,14)$. The observations of Hesselink et al. (2) are not only directly relevant to human physiology, but constitute some of the strongest data in any system addressing the basic biochemical role of UCP3 as an uncoupler. On balance, the paper seems to prove the old adage that, if you want to understand human physiology, sometimes you just have to study humans.

There are also limitations to data interpretation. The authors cannot exclude the possibility that the dietary perturbations induced some other factor that modulates an uncoupling effect of UCP3 or that an uncoupling effect was too weak to discern using this methodology, despite evidence marshaled by the authors that phosphocreatine repletion rate is sensitive to mitochondrial substrate availability. The authors also point out that UCP3 upregulation could have been counterbalanced by UCP2 downregulation (or that of other unidentified uncouplers) resulting in no net change in the mitochondrial proton leak. The authors discount this possibility for two reasons; UCP2 mRNA levels were unchanged, and other authors have provided immunological evidence that UCP2 protein is not expressed in muscle despite the presence of UCP2 mRNA (15). Even so, these latter observations were reported in rodent muscle, leaving open the possibility that UCP2 protein is present in human muscle.

\section{What is the role of UCPs?}

If the authors' conclusions are correct, then what is the biochemical role of UCP3 in humans? This question remains an intriguing and important issue for future research. Several investigators support the idea that UCP2 and UCP3 play a role in reducing reactive oxygen species formation and so protect cells from their damaging effects (16). Reactive oxygen species are produced in the course of mitochondrial respiration in a manner that is proportional to the transmembrane potential; therefore, this role would require that UCP3 function as an uncoupler. Another exciting hypothesis is that UCP3 could facilitate lipid oxidation by acting as an FFA anion transporter. Elevated circulating FFA levels are associated with increased muscle UCP3 expression in a variety of physiological states (fasting, high-fat feeding, lipid infusion, diabetes, obesity) independent of any changes in energy expenditure $(3,17,18)$. In addition, we have described an exon 6 splice donor/acceptor polymorphism in UCP3 that is associated with a marked reduction in basal lipid oxidation rates and an increase in resting respiratory quotient in Gullah-speaking African Americans (19). It is difficult to explain how an FFA anion transporter could facilitate FFA oxidation since entry of long-chain FFA through carnitine palmitoyltransferase I and subsequent oxidation requires esterification to CoA. However, under conditions of high acyl-CoA flux and lipid oxidation, accumulation of acyl-CoA would be detrimental to mitochondrial function since these molecules are strong surfactants that could damage membranes and excessive sequestration of $\mathrm{CoA}$ in the form of long chain FFA esters could inhibit $\beta$-oxidation and tricarboxylic acid cycle activity. UCPs are known to be able to export FFA anion from the mitochondrial matrix (20), but this could only help alleviate the accumulation of acyl-CoA if a thioesterase were available to remove CoA from the FFA. Recently, Moore et al. have shown that mitochondrial thioesterase- 1 is upregulated in UCP3 hyperexpressing mice and have proposed that this enzyme could perform this very function in settings of increased acyl-CoA flux (21). Thus, it is feasible to suggest that UCP3 could help sustain increased rates of lipid oxidation via export of FFA anion. In any event, as the search for the true physiological role of UCP 3 continues, confident conclusions regarding human physiology will require studies in humans.

1. Rolfe, D.F., Newman, J.M., Buckingham, J.A., Clark, M.G., and Brand, D.M. 1999. Contribution of mitochondrial proton leak to respiration rate in working skeletal muscle and liver and to SMR Am. J. Physiol. 276:C692-C699.

2. Hesselink, M.K.C., et al. 2003. Increased uncoupling protein 3 content does not affect mitochondrial function in human skeletal muscle in vivo. J. Clin. Invest. 111:479-486. doi:10.1172/JCI200316653.

3. Ricquier, D., and Bouillaud, F. 2000. The uncoupling protein homologues: UCP1, UCP2, UCP3, StUCP, and AtUCP. Biochem. J. 345:161-179.

4. Brand, M.D., Chien, L.F., Ainscow, E.K., Rolfe, D.F., and Porter, R.K. 1994. The causes and func tions of mitochondrial protein leak. Biochim. Biophys. Acta. 1187:132-139.

5. Gimeno, R.E., et al. 1997. Cloning and characterization of an uncoupling protein homologue: a potential molecular mediator of human thermogenesis. Diabetes. 46:900-906.

6. Fleury, C., et al. 1997. Uncoupling protein-2: a novel gene linked to obesity and hyperinsulinemia. Nat. Genet. 15:269-272.

7. Boss, O., et al. 1997. Uncoupling protein-3: a new member of the mitochondrial carrier family with tissue-specific expression. FEBS Lett. 408:39-42. 
8. Vidal-Puig, A., Solanes, G., Grujic, D., Flier, J.S., and Lowell, B.B. 1997. UCP3: an uncoupling protein homologue expressed preferentially and abundantly in skeletal muscle and brown adipose tissue. Biochem. Biophys. Res. Commun. 235:79-82.

9. Harper, M.E., and Himms-Hagen, J. 2001. Mitochondrial efficiency: lessons learned from transgenic mice. Biochim. Biophys. Acta. 1504:159-172.

10. Daleh, M.C., Wheeler, M.B., and Chan, C.B. 2002. Uncoupling protein 2: evidence for its function as a metabolic regulator. Diabetologia. 45:174-187.

11. Harper, J.A., et al. 2002. Artifactual uncoupling by uncoupling protein 3 in yeast mitochondria at the concentration found in mouse and rat skeletal-muscle mitochondria. Biochem. J. 361:49-56.

12. Clapham, J.C., et al. 2000. Mice overexpressing human uncoupling protein 3 in skeletal muscle are hyperphagic and lean. Nature. 406:415-418.
13. Kennedy, A., et al. 1997. The metabolic significance of leptin in humans: gender-based differences in relationship to adiposity, insulin sensitivity and energy expenditure. J. Clin. Endocrinol. Metab. 82:1293-1300.

14. Rosenbaum, M., Murphy, E.M., Heymsfield, S.B., Matthews, D.E., and Leibel, R.L. 2002. Low dose leptin administration reverses effects of sustained weight-reduction on energy expenditure and circulating concentrations of thyroid hormones. J. Clin. Endocrinol. Metab. 87:2391-2394.

15. Pecqueur, C., et al. 2001. Uncoupling protein 2 , in vivo distribution, induction upon oxidative stress, and evidence for translational regulation. J. Biol. Chem. 276:8705-8712.

16. Brand, M.D. 2000. Uncoupling to survive? The role of mitochondrial inefficiency in ageing. Exp. Gerontol. 35:811-820.

17. Bao, S., et al. 1998. Expression of mRNAs encoding uncoupling proteins in human skeletal muscle: effects of obesity and diabetes. Diabetes. 47:1935-1940.

18. Chung, W.K., et al. 1999. Genetic and physiological analysis of the role of UCP3 in energy homeostasis. Diabetes. 48:1890-1895.

19. Argyropoulos, G., et al. 1988. Effects of mutations in the human uncoupling protein 3 gene on the respiratory quotient and fat oxidation in severe obesity and type 2 diabetes. J. Clin. Invest. 102:1345-1351.

20. Garlid, K.D., Jaburek, M., and Jezek, P. 2001 Mechanism of uncoupling protein action. Biochem. Soc. Trans. 29:803-806.

21. Moore, G.B.T., Himms-Hagen, J., Harper, M.E. and Clapham, J.C. 2001. Overexpression of UCP3 in skeletal muscle of mice results in increased expression of mitochondrial thioesterase mRNA Biochem. Biophys. Res. Commun. 283:785-790. 